\title{
The role of lumboperitoneal shunts in managing chronic hydrocephalus with slit ventricles
}

\author{
Neena I. Marupudi, MD, MS, ${ }^{1}$ Carolyn Harris, PhD,, ${ }^{1,2}$ Tanya Pavri, BA, ${ }^{1}$ Brenna Mell, PA-C, ${ }^{1}$ \\ Rasanjeet Singh, BA, ${ }^{1}$ Steven D. Ham, DO, ${ }^{1}$ and Sandeep Sood, MD'1
}

\begin{abstract}
1Departments of Neurosurgery and Pediatric Neurosurgery, Wayne State University School of Medicine and Children's Hospital of Michigan, Detroit; and 'Department of Chemical Engineering and Materials Science, Wayne State University College of Engineering, Detroit, Michigan
\end{abstract}

\begin{abstract}
OBJECTIVE Lumboperitoneal (LP) shunts have a role not only in pseudotumor cerebri, but also in patients with slit-like ventricles who are treated with CSF shunting on a chronic basis. Hesitation to utilize LP shunts is based on previous conventional beliefs including the tendency for overdrainage, difficulties accessing the shunt to tap or revise, and risk of progressive cerebellar tonsillar herniation. The authors hypothesized that the use of horizontal-vertical (HV) valves may reduce the risk of these complications, particularly overdrainage and development of Chiari malformation.
\end{abstract}

METHODS All pediatric cases involving patients treated with an LP shunt at the Children's Hospital of Michigan were reviewed in this retrospective case series. A total of 143 patients with hydrocephalus were treated with LP shunts from 1997-2015 (follow-up range 8 months-8 years, median 4.2 years). Patients with pseudotumor cerebri underwent placement of an LP shunt as a primary procedure. In patients with slit ventricles from chronically treated hydrocephalus or repeated shunt malfunctions from proximal catheter obstruction, a lumbar drain was inserted to assess candidacy for conversion to an LP shunt. In patients who tolerated the lumbar drain and demonstrated communication of the ventricles with the spinal cisterns, treatment was converted to an LP shunt. All patients included in the series had undergone initial shunt placement between birth and age 16 years.

RESULTS In $30 \%$ of patients ( $n=43)$, LP shunts were placed as the initial shunt treatment; in $70 \%$ ( $n=100)$, treatment was converted to LP shunts from ventriculoperitoneal (VP) shunts. The patients' age at insertion of or conversion to an LP shunt ranged from 1 to 43 years (median 8.5 years). Of the patients with clear pre-LP and post-LP shunt follow-up imaging, none were found to develop an acquired Chiari malformation. In patients with pre-existing tonsillar ectopia, no progression was noted on follow-up MRIs of the brain in these patients after LP shunt insertion. In our LP shunt case series, no patient presented with acute deterioration from shunt malfunction.

CONCLUSIONS Conversion to an LP shunt may minimize acute deterioration from shunt malfunction and decrease morbidity of repeated procedures in patients with chronically shunt-treated hydrocephalus and small ventricles. In comparison to previously published case series of LP shunt treatment, the use of LP shunts in conjunction with HV valves may decrease the overall risk of cerebellar tonsillar herniation. The use of an LP shunt may be an alternative in the management of slit ventricles when VP shunting repeatedly fails.

https://thejns.org/doi/abs/10.3171/2018.6.PEDS17642

KEYWORDS hydrocephalus; lumbar shunt; shunt malfunction; slit ventricle syndrome; LP shunt; Chiari malformation

$\mathrm{L}$ UMBOPERITONEAL (LP) shunts are routinely used in the treatment of pseudotumor cerebri. LP shunts have also been useful in the treatment of chronically shunt-treated patients with slit-like ventricles. ${ }^{11,13,18,20}$ Shunt malfunction in patients with hydrocephalus and slitlike ventricles is challenging to both diagnose and treat. An acutely ill child with a shunt malfunction and slit-like ventricles can be at risk of death from elevated intracranial pressure, especially if the malfunction is not recognized due to the lack of ventricular size changes on brain imaging. ${ }^{25}$ LP shunts in this patient population can significantly reduce the rate and severity of acute deterioration from shunt malfunction. ${ }^{20}$

Many neurosurgeons' hesitation to utilize an LP shunt

ABBREVIATIONS CSF = cerebrospinal fluid; EAM = external auditory meatus; $E V D=$ external ventricular drain; $H V=$ horizontal-vertical; $I Q R=$ interquartile range; $L P=$ lumboperitoneal; $\mathrm{VP}=$ ventriculoperitoneal.

SUBMITTED November 17, 2017. ACCEPTED June 6, 2018.

INCLUDE WHEN CITING Published online September 21, 2018; DOI: 10.3171/2018.6.PEDS17642. 
in the treatment of hydrocephalus is based on previous conventional beliefs. These include the tendency for overdrainage, difficulty in accessing an LP shunt for cerebrospinal fluid (CSF) sampling, programmable valve changes, or shunt revision, and a reported higher risk of subdural hematoma formation in the adult population. . $^{1,12}$ Previous publications have reported low-pressure headaches and cerebellar tonsillar herniation as sequelae of overdrainage from LP shunts. . $^{, 6,17}$

In 1993, Chumas et al. reported radiographic evidence of cerebellar tonsillar herniation in a large percentage of patients who had undergone insertion of LP shunts. ${ }^{5}$ In these patients, the LP shunt components included a straight catheter or T-shunt with a distal slit valve. With the exception of this study, no other large series of patients treated with LP shunts with long-term follow-up has been reported. In 2007, Wang et al. reported on the utility of the horizontal-vertical (HV) valve and its efficacy in reducing the incidence of overdrainage. ${ }^{24}$ However, no large series has reported long-term outcomes with the use of an $\mathrm{HV}$ valve for LP shunts and the incidence of Chiari malformation development. Because $\mathrm{HV}$ valve systems offer higher resistance in the upright position, they minimize overdrainage in patients with LP shunts. As a reassessment of the utility and safety of LP shunts, we report on the Children's Hospital of Michigan experience with LP shunt treatment and long-term outcomes.

\section{Methods \\ Patient Population}

After obtaining approval from the Wayne State University Institutional Review Board, we performed a retrospective analysis of all cases in which patients underwent LP shunt placement or revision at the Children's Hospital of Michigan, Detroit, between 1997 and 2015. Through analysis of the operating room records, we identified 143 patients for inclusion in our case series. Each patient's demographic data, diagnosis, type of shunt, dates and types of operations, intraoperative findings, shunt hardware used, and imaging results were analyzed for development of Chiari malformations and subdural hematomas. Symptoms of shunt malfunction presentation, if applicable, were also included in the analysis. Preoperative imaging and subsequent imaging were also screened, up to the patients' most recent follow-up, to assess for the presence or development of a Chiari malformation. The patients' age at initial shunt placement (ventriculoperitoneal [VP] or LP shunt) ranged from birth to 16 years. Their age at conversion to LP shunt treatment ranged from 3 to 27 years.

Patients with pseudotumor cerebri requiring CSF diversion were considered candidates for LP shunt placement as an initial shunt. Patients with the combination of slit-like ventricles from chronic shunting and repeated shunt malfunctions related to proximal catheter obstruction were considered candidates for conversion to an LP shunt. The duration of follow-up ranged from 8 months to 8 years (median 4.2 years).

\section{Evaluation for Chiari Malformation}

Sagittal images, either MRI or reconstructed CT imag- es, were reviewed to assess for Chiari malformation in all patients who had undergone treatment with an LP shunt. Chiari measurements were performed by measuring the vertical distance from the tip of the cerebellar tonsils to a line drawn between the anterior and posterior margins of the foramen magnum. For this study, patients with less than $5 \mathrm{~mm}$ of tonsillar descent were defined as having tonsillar ectopia, and those with more than $5 \mathrm{~mm}$ of tonsillar ectopia were defined as having a Chiari malformation. Progression in Chiari malformation was defined as an interval increase in the vertical distance on subsequent imaging. After insertion of an LP shunt, patients were followed with serial brain MRI, or CT if unable to tolerate MRI. An initial follow-up scan was done 6 months to 1 year after the procedure, and additional follow-up scans were performed every 2-3 years thereafter.

\section{Procedural Steps and Considerations for Conversion to LP Shunt}

Once a patient was determined to be a potential candidate for LP shunt insertion, the VP shunt was externalized to an external ventricular drain (EVD) in the operating room, and a lumbar drain was also subsequently inserted. The EVD and lumbar drains were initially managed by leveling each respective transducer to the patient's external auditory meatus (EAM) and setting both the EVD and the lumbar drain to drain at $0 \mathrm{~cm} \mathrm{H}_{2} \mathrm{O}$. Over the following few days, the lumbar drain was maintained at the level of the EAM while the level of the EVD was gradually raised over 2-3 days (e.g., lumbar drain at $0 \mathrm{~cm} \mathrm{H}_{2} \mathrm{O}$, while EVD at $10 \mathrm{~cm} \mathrm{H}_{2} \mathrm{O}$ above the EAM) to bias drainage toward the lumbar system. A CT ventriculogram was performed to assess for sites of fluid obstruction. Dye was injected through the EVD prior to imaging, and if no obstruction into the cervical cisterns was evident, then the patient was determined to be a candidate for an LP shunt (Fig. 1). If aqueductal obstruction was identified on the CT ventriculogram, then an endoscopic third ventriculostomy was considered. Following third ventriculostomy in these patients, both of the drains were clamped to assess the possibility of shunt independence. Patients who were unable to tolerate this clamping were considered candidates for an LP shunt. As a last step prior to placement of the LP shunt, the EVD was clamped while the lumbar drain remained unclamped and the patient's condition was monitored to ensure that lumbar drainage alone would be adequate. We have also included preoperative MRI of the brain as part of our standardized protocol, prior to insertion of an LP shunt, in order to evaluate for and document any pre-existing tonsillar ectopia or Chiari malformation.

For insertion of the LP shunt, the patient was positioned in the operating room in the lateral position, supported by a beanbag, with the back, right flank, and right side of the abdomen exposed. Access to the lumbar cistern was obtained with a Tuohy needle, through which a lumbar catheter was fed into the spinal cistern. Once an adequate length of the catheter was inserted, the needle was removed. Peritoneal access was obtained via a minilaparotomy or split trocar access. A tunneler was passed from the abdominal incision to the lumbar incision. If needed, a small transverse incision was made at the right 

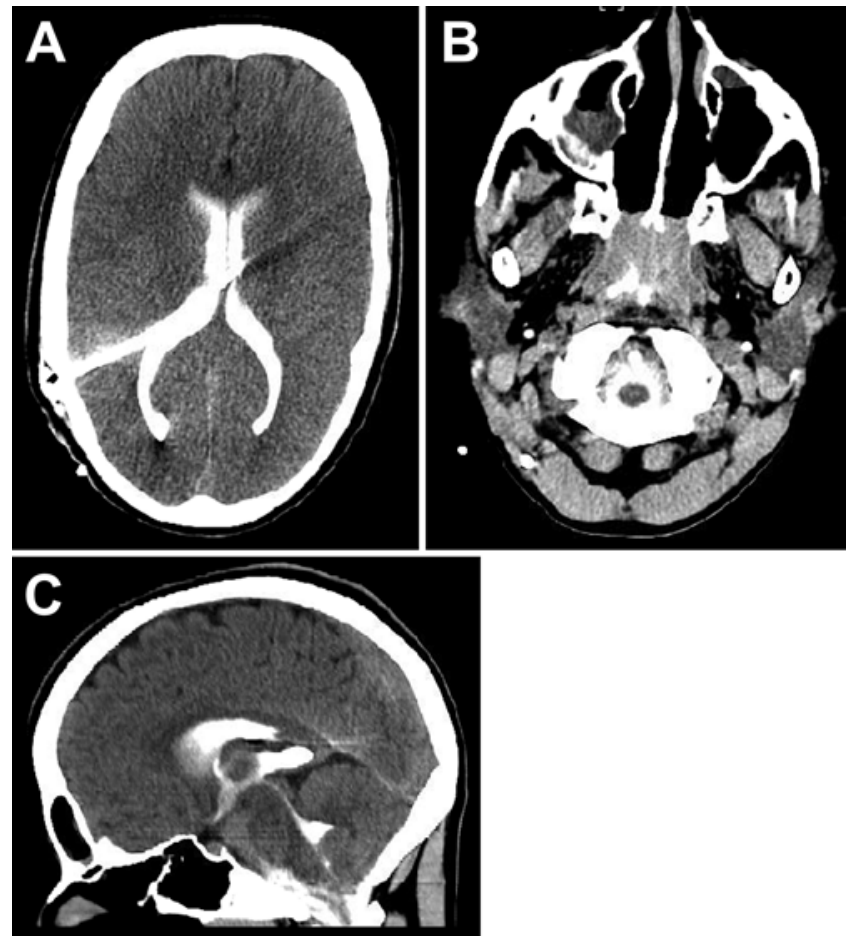

FIG. 1. CT ventriculogram, dye-injection study, confirming communication between ventricles and spinal subarachnoid space. Dye was administered via reservoir or catheter into the ventricle. A: Axial view of ventricular system with dye throughout the system. B: Axial view demonstrating dye at the foramen magnum and lower brainstem. C: Sagittal view demonstrating dye throughout ventricular system.

flank for tunneling. An HV valve was selected based on patient's height, ventricular size, and desired pressure. The valve was placed either at the flank incision or at an extended abdominal incision. Once the lumbar catheter was connected to the valve and the valve was connected to the peritoneal tubing with confirmation of adequate CSF flow, the peritoneal catheter was inserted into the peritoneal space. We sutured the HV valve in place, with a 3 -point fixation to the subcutaneous tissue, to ensure that it would maintain its orientation (Fig. 2). The EVD was then removed and replaced with a ventricular reservoir for future assessment of intracranial pressure or patency of third ventriculostomy at later presentations with suspected malfunction.

\section{Results}

One hundred forty-three patients were identified as having had an LP shunt placed or revised from 1997 to 2015 (82 male and 61 female patients). The majority of these patients had acquired hydrocephalus $(\mathrm{n}=88,61.5 \%$ of patients) related to a variety of etiologies, including intraventricular hemorrhage of prematurity and pseudotumor cerebri. The remaining patients presented with congenital hydrocephalus ( $n=55,38.5 \%$ of patients). Forty-three patients (30.1\%) underwent initial shunting with an LP shunt for conditions commonly managed with lumbar shunts (e.g., pseudotumor cerebri). In the rest $(n=100,69.9 \%)$ of the cases identified for this series, a ventricle-based shunt was converted to an LP shunt. Patients selected for shunt conversion to an LP shunt were specifically patients with repeated shunt malfunctions, small ventricles, and concerns for insufficient management of their hydrocephalus with a ventricle-based shunt system. The mean age at initial shunt placement (LP or VP) was $3.7 \pm 5.6$ years. The mean age at initial LP shunt placement was $10.0 \pm 7.2$ years (median age 8.5 years at LP shunt placement, range 1-43 years, interquartile range [IQR] 4.1-15.3 years), and the mean duration for which these patients had an LP shunt was $7.6 \pm 5.0$ years (range 0.05-32.7 years) (Table 1). $\mathrm{HV}$ valves were used in all patients with LP shunts.

Of the 143 patients in the series, 31 had imaging-based documentation of tonsillar ectopia or Chiari type I mal-
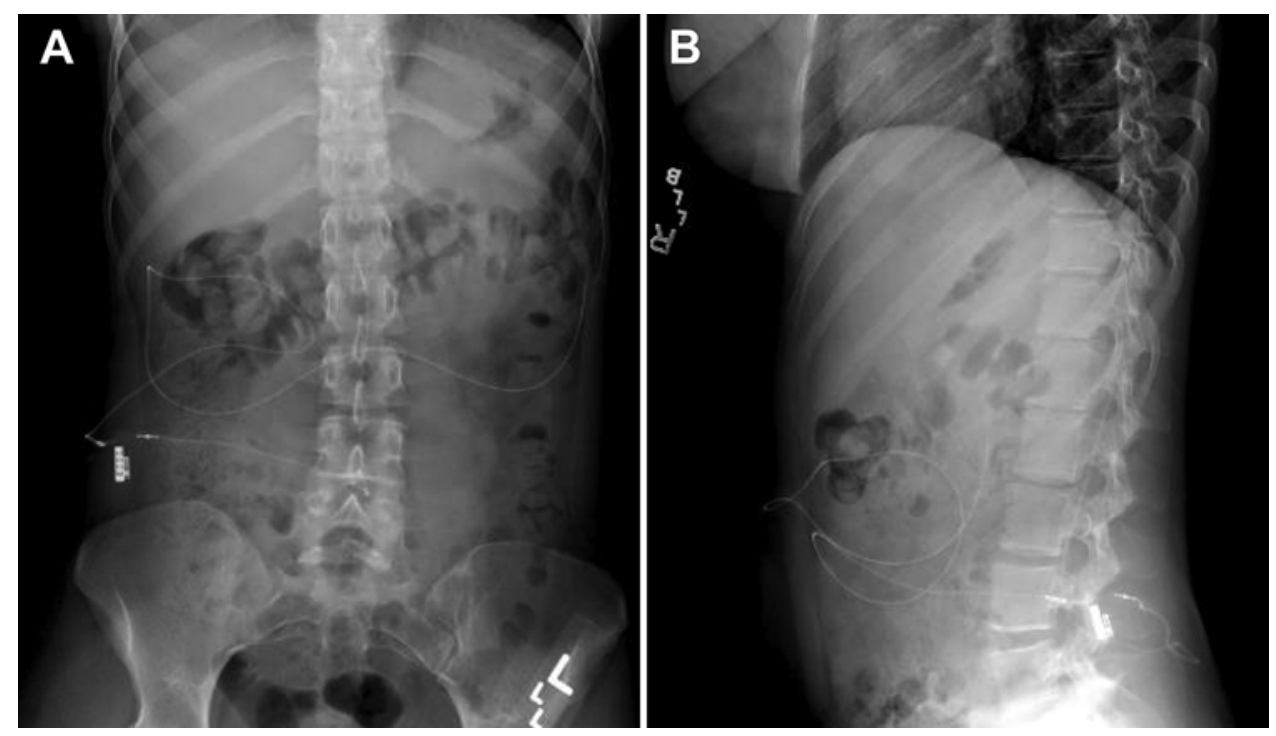

FIG. 2. Radiographs for LP shunt series. Anterior-posterior view $(\mathbf{A})$ and lateral view $(B)$ radiographs demonstrating continuity of LP shunt system, including lumbar spinal catheter, HV valve, and peritoneal catheter. 
TABLE 1. Summary of clinical and demographic characteristics of 143 patients with a history of LP CSF shunting

\begin{tabular}{|c|c|}
\hline Characteristic & Value \\
\hline Total no. of patients & 143 \\
\hline \multicolumn{2}{|l|}{ Age at last follow-up (yrs) } \\
\hline Median & 20.3 \\
\hline IQR & $12.8-23.6$ \\
\hline \multicolumn{2}{|l|}{ Sex } \\
\hline Male & $83(58 \%)$ \\
\hline Female & $61(52 \%)$ \\
\hline \multicolumn{2}{|l|}{ Etiology } \\
\hline Acquired & $88(61.5 \%)$ \\
\hline Congenital & $55(38.5 \%)$ \\
\hline \multicolumn{2}{|l|}{ 1st shunt type } \\
\hline VP & $98(68.5 \%)$ \\
\hline LP & $43(30.1 \%)$ \\
\hline VA & $2(1.4 \%)$ \\
\hline \multicolumn{2}{|l|}{ Age at conversion to LP shunt* (yrs) } \\
\hline Median & 8.5 \\
\hline IQR & $4.1-15.3$ \\
\hline \multicolumn{2}{|l|}{ Primary reason for LP shunt placement } \\
\hline Slit-like or small ventricles (+ shunt revisions) & $75(52.4 \%)$ \\
\hline Multiple shunt revisions & $30(21 \%)$ \\
\hline Pseudotumor cerebri & $38(26.6 \%)$ \\
\hline
\end{tabular}

$\mathrm{VA}=$ ventriculoatrial.

Data are numbers of patients (\%) unless otherwise indicated.

${ }^{*}$ Age at the time of LP shunt placement for patients who underwent conversion from ventricle-based shunt treatment to LP shunt treatment, excluding patients who received an LP shunt as initial CSF shunt treatment.

formation. Of those 31 patients, 16 had documented tonsillar ectopia or Chiari malformation on MRI prior to insertion of an LP shunt. While the remaining 15 patients did not undergo MRI prior to shunting, review of preoperative CT scans demonstrated crowding at the foramen magnum consistent with either tonsillar ectopia or Chiari malformation. All LP shunt-treated patients identified with tonsillar ectopia or Chiari malformation, were followed closely with periodic imaging. No progression of the tonsillar ectopia or Chiari malformation was noted on follow-up imaging in these patients. In addition, none of the patients developed clinical symptoms concerning for obstructive CSF flow from their incidentally identified Chiari malformations. Patients believed to be clinically symptomatic from a Chiari malformation were not considered for conversion to an LP shunt.

In our series, none of the patients treated with LP shunts developed subdural hematomas. Upon reviewing all clinical presentations of incidence of shunt malfunction in the LP shunt-treated patients, we found that no patient with an LP shunt presented with acute neurological deterioration from shunt malfunction. Patients treated with a VP shunt who presented in extremis from ventricular shunt malfunctions $(\mathrm{n}=14)$ were followed after conversion to LP shunt. The majority ( 8 of 14 ) did not require further revisions. While 6 of 14 required further revisions, none presented in extremis after conversion to an LP shunt.

\section{Discussion}

Progressive loss of interstitial fluid through the venous system, as a result of increasing pressure within the ventricles, is responsible for ventricular enlargement in patients with hydrocephalus. However, in some patients who have been treated with chronic CSF shunting, the ventricles remain small despite a high pressure. In patients who have undergone chronic CSF shunting, studies have demonstrated the presence of periventricular gliosis. ${ }^{7-10,14}$ One theory is that periventricular gliosis can restrict ventricles from dilating, and when the shunt malfunctions, the patient can present with a hypertensive slit ventricle syndrome. In another study, in patients with shunt malfunction in whom the ventricles had failed to dilate, a differential pressure gradient was not found across the periventricular region with a bolus injection into the ventricle. This finding combined with the simultaneous measurement of pressure from the ventricle and periventricular parenchyma suggests that periventricular gliosis may not be the mechanism restricting ventricular dilatation..$^{22}$ Our previous studies of patients with slit-like ventricles have suggested that perhaps these patients actually have a high and not low compliance..$^{20,22}$ Since the brain itself is inelastic and intracranial compliance could be due to the compliance of the veins, this group of patients, therefore, may have a high intracranial venous compliance, potentially due to chronic CSF overdrainage and resultant increase in the intracranial venous pool.

The increase in the intracranial venous pool has been observed on imaging studies as venous engorgement and indirectly inferred from pressure-volume index studies. ${ }^{2,3,16,21}$ Increased venous engorgement that results from chronic CSF overdrainage makes veins larger but also prone to rapid collapse with slight changes in transmural pressure from a malfunctioning shunt. As a result of this acute venous obstruction, the ventricles may not enlarge in patients who have been treated with chronic CSF shunting, and these patients may present acutely with severe headaches, bradycardia, sometimes seizures, and persistent small ventricles. This conceptual framework is supported by the observation that ventricles in these patients can enlarge if intracranial pressure is reduced by draining CSF from the spinal subarachnoid space. ${ }^{22,23}$ The reduction in intracranial pressure opens up the draining veins and restores venous drainage. This allows movement of interstitial fluid into the venous system, resulting in enlargement of ventricles.

In addition to the physiological changes described above, patients who are treated with ventricular shunts on a chronic basis can develop anatomical changes in the ventricular system, necessitating insertion of multiple catheters because of ventricular isolation. These anatomical changes result from reversal of normal flow of CSF from the lateral to the third and then the fourth ventricle. The reversal in flow between the lateral and third ventricles initiates progressive closure of the foramen of Monro, and the reversal between the third and fourth ventricles results in secondary aqueductal obstruction. Likewise, a parietal 
ventricular catheter can cause reversal of flow from the temporal horn to the frontal horn, resulting in isolation of the body and atrium of the lateral ventricle from the temporal horn. ${ }^{20}$

Patients with small slit-like ventricles are also prone to repetitive malfunctions from crowding of the choroid plexus around the ventricular catheter and consequent ingrowth and recurrent proximal obstruction. Repeated revisions in the face of small ventricles can predispose this group of patients to infections, catheter-related strokes, and intracranial hemorrhage. ${ }^{4}$

Our case series of LP shunt-treated patients shows that a lumbar shunt may mitigate some of these problems in patients who previously had a ventricular shunt. Since the lumbar shunt catheter is intraspinal and not in close proximity to the choroid plexus, the rate of proximal malfunction is significantly reduced. ${ }^{20}$ Furthermore, there is a lower risk of stroke or intracranial hemorrhage related to revisions.

More importantly, while some patients with VP shunts and slit-like ventricles would present with acute neurological deterioration with shunt malfunction, patients whose VP shunts had been converted to LP shunts did not present with acute or severe neurological deterioration. This reduction in acute deterioration may be related to improvement in intracranial venous compliance that occurs over time after conversion to an LP shunt. With ventricular shunts the effect of negative pressure is on the intracranial venous system, whereas the effect of negative pressure generated by a lumbar shunt is likely to affect the spinal epidural venous plexus in its immediate vicinity, changing its compliance. Hence, acute lumbar shunt malfunction may cause rapid collapse of the spinal venous epidural plexus, and as a result, does not result in the acute pseudotumor-like presentation that accompanies ventricular shunt malfunction as described above. In addition, spinal arachnoid villi in contact with the epidural venous plexus may also be a conduit for CSF absorption; arachnoid villi in lumbosacral nerve roots increase CSF absorption in the upright position in response to gravity. ${ }^{19}$
Patients with LP shunts may present with silent papilledema at the time of lumbar shunt malfunction. In our series, all patients who had their ventricular shunt successfully converted to a lumbar shunt had communicating CSF pathways and open subarachnoid spaces. Hence, at the time of malfunction, egress of CSF through the open optic nerve sheaths or the spinal nerve sheaths may have contributed to maintaining a high normal CSF pressure but resulted in development of papilledema. As a policy now, all patients with a lumbar shunt are followed with a funduscopic examination twice a year at our institution.

Despite certain advantages, the use of lumbar shunts in pediatric patients remains limited due to concern over the potential development of Chiari malformation over time from negative suction effect, highlighted by Chumas et al. in 1993. 5,6 At that time, these authors reported their results with the use of a valveless shunt or shunt with only slit valves. On the other hand, our series demonstrates that with the use of an HV valve, an LP shunt does not necessarily put a patient at increased risk for development or worsening of radiologically identified Chiari malformation. In our series, long-term clinical and radiographic follow-up of LP shunt-treated patients without a preoperative Chiari malformation has not, thus far, resulted in the development of tonsillar herniation, even years after placement of an LP shunt that incorporates an HV valve. Patients treated with LP shunts who had a history of preoperative radiographic Chiari type I malformation were followed closely with serial imaging; these patient did not experience Chiari symptoms or radiographic progression of the Chiari malformation on follow-up (Fig. 3).

The unusually high percentage of patients with Chiari malformations may be attributable to ventricular shunting at a young age, which not only increases the risk of hypertensive slit ventricle syndrome, but also results in inadequate expansion of the posterior fossa, thereby resulting in crowding and tonsillar herniation. This phenomenon was initially seen and described in patients with arachnoid cysts who had shunts placed at a very young age. ${ }^{15}$

Although, we present the benefits of an LP shunt in the
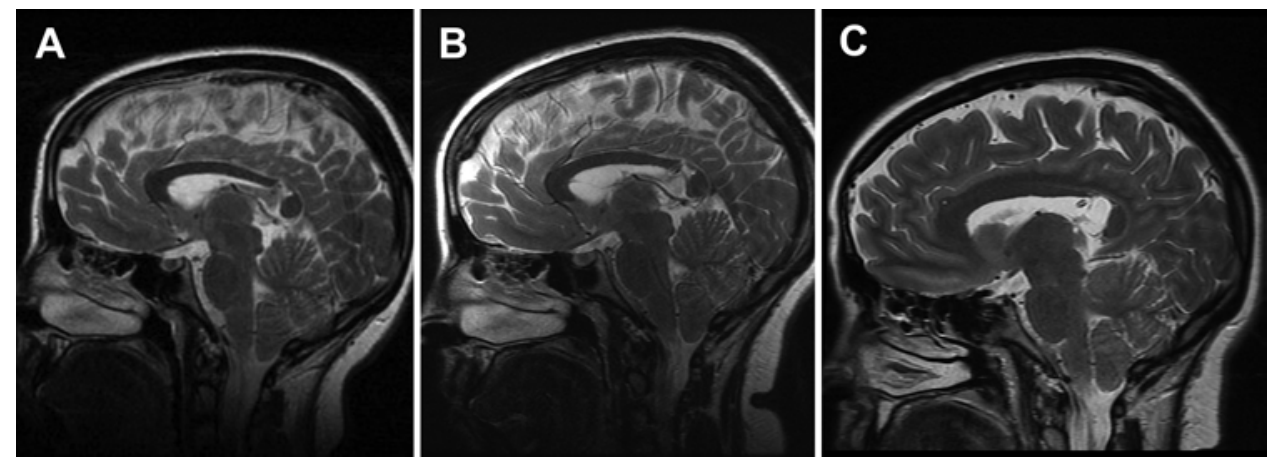

FIG. 3. MR images showing no progression of Chiari malformation in an LP shunt-treated patient. This 25 -year-old woman had a history of hydrocephalus related to meningitis as infant. A VP shunt was placed at 9 months and converted to an LP shunt when the patient was 14-years-old due to small ventricles and multiple shunt revisions of the proximal ventricular catheter. A: Sagittal T2-weighted MR image obtained prior to LP shunt placement showing $8 \mathrm{~mm}$ cerebellar tonsillar descent. The patient had no Chiari-related symptoms at the time. B: Sagittal T2-weighted MR image obtained 3 years after LP shunt placement showing no change in tonsillar descent. The patient had no Chiari-related symptoms at the time of this MRI study. C: Sagittal T2 MR image obtained 11 years after LP shunt placement showing slight improvement in tonsillar descent to $6 \mathrm{~mm}$. The patient remained free of any Chiari-related symptoms. 
specific circumstances of small ventricles and repeated proximal catheter malfunctions with a VP shunt, our results do not support nor do we recommend the use of LP shunts as a routine initial CSF diversion procedure. Further prospective case-control studies are needed to further standardize a protocol for the use of LP shunts with HV valves and also to validate the suspected benefits. Nonetheless, LP shunts should be considered in the treatment of certain types of hydrocephalus when other CSF diversion systems fail, especially in light of our large series demonstrating an improved safety profile of LP shunts with the use of $\mathrm{HV}$ valves.

\section{Conclusions}

LP shunts utilizing HV valves can safely be included in the repertoire of types of shunts used to treat certain types of hydrocephalus. Conversion to LP shunts minimizes acute deterioration from shunt malfunction. LP shunts decrease morbidity of repeat procedures in patients with chronically shunt-treated hydrocephalus and small ventricles. An increased incidence of Chiari malformations is not present with the use of HV valves for hydrocephalus patients treated with LP shunts.

\section{References}

1. Aoki N, Mizutani H: Acute subdural hematoma due to minor head trauma in patients with a lumboperitoneal shunt. Surg Neurol 29:22-26, 1988

2. Barami K, Sood S: The cerebral venous system and the postural regulation of intracranial pressure: implications in the management of patients with cerebrospinal fluid diversion. Childs Nerv Syst 32:599-607, 2016

3. Barami K, Sood S, Ham SD, Canady AI: Postural changes in intracranial pressure in chronically shunted patients. Pediatr Neurosurg 33:64-69, 2000

4. Calayag M, Paul AR, Adamo MA: Intraventricular hemorrhage after ventriculoperitoneal shunt revision: a retrospective review. J Neurosurg Pediatr 16:42-45, 2015

5. Chumas PD, Armstrong DC, Drake JM, Kulkarni AV, Hoffman HJ, Humphreys RP, et al: Tonsillar herniation: the rule rather than the exception after lumboperitoneal shunting in the pediatric population. J Neurosurg 78:568-573, 1993

6. Chumas PD, Kulkarni AV, Drake JM, Hoffman HJ, Humphreys RP, Rutka JT: Lumboperitoneal shunting: a retrospective study in the pediatric population. Neurosurgery 32:376-383, 1993

7. Del Bigio MR: Neuropathological findings in a child with slit ventricle syndrome. Pediatr Neurosurg 37:148-151, 2002

8. Del Bigio MR, Bruni JE: Periventricular pathology in hydrocephalic rabbits before and after shunting. Acta Neuropathol 77:186-195, 1988

9. Del Bigio MR, Bruni JE: Reaction of rabbit lateral periventricular tissue to shunt tubing implants. J Neurosurg 64:932-940, 1986

10. Del Bigio MR, Bruni JE, Fewer HD: Human neonatal hydrocephalus. An electron microscopic study of the periventricular tissue. J Neurosurg 63:56-63, 1985

11. Ide T, Aoki N, Miki Y: Slit ventricle syndrome successfully treated by a lumboperitoneal shunt. Neurol Res 17:440-442, 1995

12. Kamiryo T, Hamada J, Fuwa I, Ushio Y: Acute subdural hematoma after lumboperitoneal shunt placement in patients with normal pressure hydrocephalus. Neurol Med Chir (Tokyo) 43:197-200, 2003
13. Le H, Yamini B, Frim DM: Lumboperitoneal shunting as a treatment for slit ventricle syndrome. Pediatr Neurosurg 36:178-182, 2002

14. Mangano FT, McAllister JP II, Jones HC, Johnson MJ, Kriebel RM: The microglial response to progressive hydrocephalus in a model of inherited aqueductal stenosis. Neurol Res 20:697-704, 1998

15. Martínez-Lage JF, Ruíz-Espejo AM, Almagro MJ, Alfaro R, Felipe-Murcia M, López-Guerrero AL: CSF overdrainage in shunted intracranial arachnoid cysts: a series and review. Childs Nerv Syst 25:1061-1069, 2009

16. Mokri B: The Monro-Kellie hypothesis: applications in CSF volume depletion. Neurology 56:1746-1748, 2001

17. Payner TD, Prenger E, Berger TS, Crone KR: Acquired Chiari malformations: incidence, diagnosis, and management. Neurosurgery 34:429-434, 1994

18. Rekate HL, Wallace D: Lumboperitoneal shunts in children. Pediatr Neurosurg 38:41-46, 2003

19. Sakka L, Coll G, Chazal J: Anatomy and physiology of cerebrospinal fluid. Eur Ann Otorhinolaryngol Head Neck Dis 128:309-316, 2011

20. Sood S, Barrett RJ, Powell T, Ham SD: The role of lumbar shunts in the management of slit ventricles: does the slit-ventricle syndrome exist? J Neurosurg 103 (2 Suppl):119-123, 2005

21. Sood S, Kumar CR, Jamous M, Schuhmann MU, Ham SD, Canady AI: Pathophysiological changes in cerebrovascular distensibility in patients undergoing chronic shunt therapy. $\mathbf{J}$ Neurosurg 100 (5 Suppl Pediatrics):447-453, 2004

22. Sood S, Lokuketagoda J, Ham SD: Periventricular rigidity in long-term shunt-treated hydrocephalus. J Neurosurg 102 (2 Suppl):146-149, 2005

23. Vassilyadi M, Farmer JP, Montes JL: Negative-pressure hydrocephalus. J Neurosurg 83:486-490, 1995

24. Wang VY, Barbaro NM, Lawton MT, Pitts L, Kunwar S, Parsa AT, et al: Complications of lumboperitoneal shunts. Neurosurgery 60:1045-1049, 2007

25. Winston KR, Lopez JA, Freeman J: CSF shunt failure with stable normal ventricular size. Pediatr Neurosurg 42:151155,2006

\section{Disclosures}

The authors report no conflict of interest concerning the materials or methods used in this study or the findings specified in this paper.

\section{Author Contributions}

Conception and design: Sood, Marupudi, Ham. Acquisition of data: Marupudi, Pavri, Mell. Analysis and interpretation of data: Sood, Marupudi, Harris, Singh. Drafting the article: Marupudi. Critically revising the article: Sood, Marupudi, Harris, Mell. Reviewed submitted version of manuscript: all authors. Approved the final version of the manuscript on behalf of all authors: Sood. Statistical analysis: Marupudi. Administrative/technical/material support: Sood, Marupudi. Study supervision: Sood, Marupudi.

\section{Supplemental Information \\ Previous Presentations}

This paper was presented as a platform presentation at the 84th Annual Meeting of American Association of Neurological Surgeons, April 30-May 6, 2016 in Chicago, IL.

\section{Correspondence}

Sandeep Sood: Wayne State University School of Medicine, Children's Hospital of Michigan, Detroit, MI. ssood@med.wayne.edu. 